\title{
DESENVOLVIMENTO DE EQUIPAMENTO DE BAIXO CUSTO PARA DETERMINAÇÃO DE TAMANHO DE POROS E MOLHABILIDADE DE MATERIAIS POROSOS
}

\author{
Gabriel Felipe Rocha ${ }^{1}$, Laíse M. Furtado ${ }^{1}$, Denise F. S. Petri ${ }^{1, *}$ \\ 1 -Instituto de Química, Universidade de São Paulo (USP), São Paulo, SP \\ dfsp@usp.br
}

\begin{abstract}
Resumo
Neste trabalho foi desenvolvido um equipamento de baixo custo para determinar o tamanho médio de poros através do fenômeno de ascensão capilar. Medidas com sorção de n-hexano ou n-dodecano permitiram determinar o raio médio de filtros de papel comerciais e máscara cirúrgica descartável através da equação de Washburn. A partir do tamanho médio dos poros e de medidas de sorção de água foi possível determinar o ângulo de contato entre água e os papéis de filtro. Espera-se que alunos dos cursos de graduação do IQ- USP façam uso do aparelho construído neste trabalho, estudando diversos conceitos, tais como molhabilidade, interações intermoleculares, tensão superficial, capilaridade, e o método de Washburn.
\end{abstract}

Palavras-chave: molhabilidade, Washburn, Arduino, poros

Development of low cost equipment for determining pore size and wettability of porous materials

\begin{abstract}
:
In this work, a low-cost equipment was constructed to determine the mean pore size by the capillary rise. Measurements of n-hexane or n-dodecane sorption allowed determining the mean pore size of commercial filter paper and disposable masks, by means of Washburn equation. From the mean pore size and measurements of water sorption, it was possible to determine the contact angle between water and filter papers. IQ-USP undergraduate students are expected to make use of the device built in this work, studying several concepts, such as wettability, intermolecular interactions, surface tension, capillarity, and the Washburn method.
\end{abstract}

Keywords: wettability, Washburn, Arduino, pores.

\section{Introdução}

A molhabilidade é um conceito relacionado à facilidade de um líquido se espalhar sobre uma superfície. A molhabilidade de uma superfície depende do balanço das forças coesivas do líquido e forças adesivas entre o líquido e a superfície ${ }^{1,2}$. A equação de Young relaciona o ângulo de contato $(\theta)$ entre uma gota de líquido e uma superfície sólida homogênea e plana no equilíbrio ${ }^{1,2}$ :

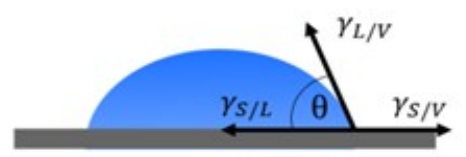

$$
\cos \theta=\frac{\gamma_{S / V}-\gamma_{S / L}}{\gamma_{L / V}}
$$

onde $\gamma_{\mathrm{S} / \mathrm{L}}, \gamma_{\mathrm{L} / \mathrm{V}}$ e $\gamma_{\mathrm{S}} / \mathrm{v}$ correspondem à tensão interfacial entre líquido e sólido, tensão superficial do líquido e energia superficial do sólido, respectivamente.

Valores de $\theta$ menores que $90^{\circ}$ indicam molhabilidade espontânea, acima deste valor o líquido não molha o sólido, já em ângulo nulo o líquido molha completamente o sólido. Quanto mais favoráveis forem as interações entre as moléculas do líquido e o sólido menor será o valor de $\theta^{1,2}$. 
O método da gota séssil não é adequado para medir $\theta$ de materiais porosos, porque os poros ficam preenchidos por ar, alterando o valor de $\theta$. As medidas de ângulo de contato de materiais porosos devem ser feitas pelo método de sorção. A sorção de líquidos por materiais porosos envolve o fenômeno de ascensão capilar. Esse fenômeno é muito importante no transporte de seiva pelos finos capilares nas plantas e pode ser facilmente observado ao introduzir capilar fino de vidro em um recipiente contendo água. A água molha bem o vidro devidos às ligações de $\mathrm{H}$ com os grupos silanóis da superfície do vidro. Nestas situações, as forças adesivas são maiores que as forças coesivas. Se o raio (r) do capilar de vidro é pequeno, haverá a formação de um menisco curvo. Laplace mostrou que a pressão do lado côncavo ( $\mathrm{P}_{\text {côncavo }}$ ) de uma interface curva é sempre maior que a do lado convexo $\left(\mathrm{P}_{\text {convexo }}\right)$ e esta diferença de pressão depende da tensão superficial do líquido $\left(\gamma_{\mathrm{L} / \mathrm{V}}\right)$ e do ângulo de contato entre a parede do capilar e do líquido ${ }^{1,2}$ :

$$
P_{\text {côncavo }}-P_{\text {convexo }}=\frac{2 \gamma_{L / V}}{r} \cos \theta
$$

A diferença de pressão é compensada pela pressão hidrostática da coluna de líquido de altura $\Delta \mathrm{h}$ :

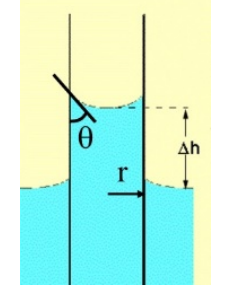

$$
\Delta h \times \rho \times g=\frac{2 \gamma}{r} \cos \theta
$$

onde $\rho$ e g são a densidade do líquido e a aceleração da gravidade, respectivamente.

A equação de Washburn deriva da combinação da equação de Laplace (eq. 2) (forças capilares) e da equação de Poiseuille (forças viscosas) para um fluido incompressível em fluxo laminar através de um cilindro de seção transversal de raio $\mathrm{r}$ constante ${ }^{1,2}$ :

$$
\frac{h^{2}}{t}=\frac{r \gamma \cos \theta}{2 \eta}
$$

onde h é altura da coluna de líquido que subiu por capilaridade em um dado período de tempo t e $\eta$ é a viscosidade do líquido.

\section{Experimental}

O equipamento foi desenvolvido com peças de Lego ${ }^{\circledR}$, placa Arduino ${ }^{\circledR}$ e peças finas de madeira (Figura 1a). As análises foram realizadas com água destilada, n-hexano e dodecano fornecidos pela Labsynth. O equipamento foi utilizado para caracterizar papel de filtro com gramatura de $80 \mathrm{~g} / \mathrm{m}^{2} \mathrm{e}$ $0,2 \mathrm{~mm}$ de espessura, e outro com gramatura de $250 \mathrm{~g} / \mathrm{m}^{2}$ e espessura de $0,5 \mathrm{~mm}$, e a camada interna de máscara hospitalar de polipropileno (Figura 1b). As amostras foram cortadas em tiras de $10 \mathrm{~cm}$ de altura por $1 \mathrm{~cm}$ de largura e $10 \mathrm{~cm}$ de altura e $0,5 \mathrm{~cm}$ de largura para avaliar o efeito da área da fita de papel na ascensão capilar. Em seguida, as amostras foram fixadas na presilha, o líquido foi adicionado no copo dosador e posicionado na plataforma elevatória. A câmera do celular foi posicionada para a gravação da ascensão capilar, que durou $\sim 90$ s. Para a edição do vídeo foi utilizado 
o software Windows Movie Maker (Microsoft), ele foi dividido em 20 frames, que foram convertidos em imagens pelo Free video to jpg converter. Com as imagens foram obtidos dados de $\mathrm{h}$ em função do tempo (t). Todos os ensaios foram realizados em duplicata, na temperatura de $23 \pm 1{ }^{\circ} \mathrm{C}$.

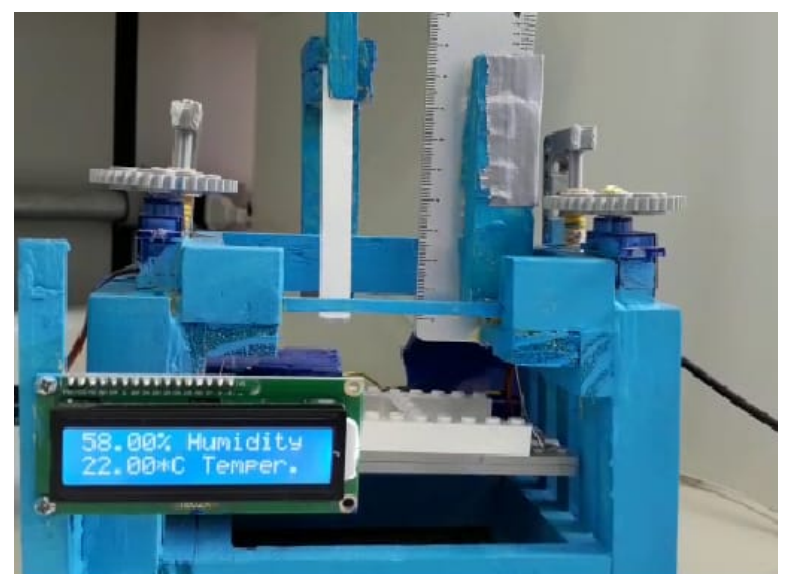

(a)

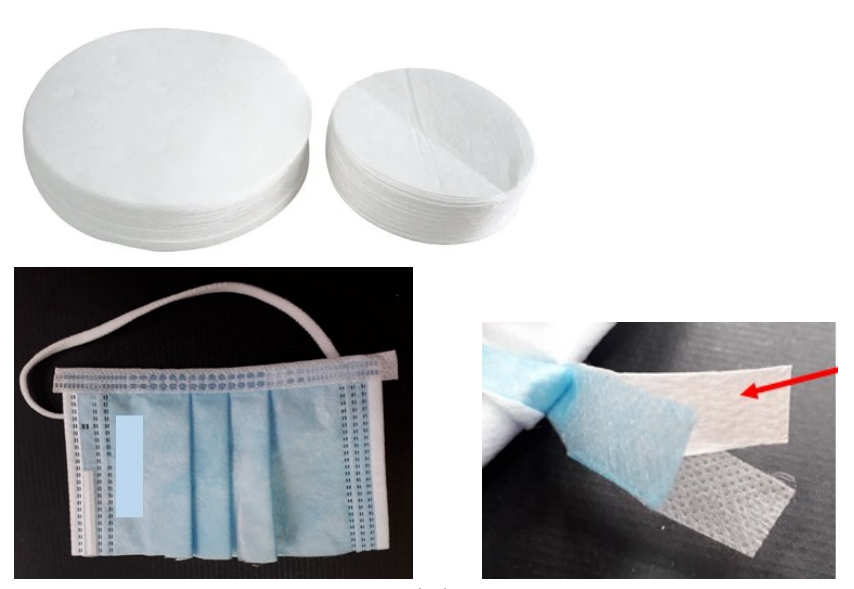

(b)

Figura 1. Fotografia (a) do equipamento automatizado desenvolvido com peças de Lego®, placa Arduino ${ }^{\circledR}$ e peças finas de madeira e (b) dos materiais porosos investigados.

Primeiramente, foram realizados ensaios com n-hexano $\left(\theta=0^{\circ}\right)$ e n-dodecano $\left(\theta=0^{\circ}\right)$, para estimar o raio médio (r) dos poros através da equação (4). Em seguida, foram realizadas medidas com água para determinação de $\theta$. Análises de microscopia eletrônica de varredura (MEV) foram realizadas para os três materiais recobertos com ouro $(\sim 5 \mathrm{~nm})$ no microscópio Jeol Neoscope JCM-5000 operando com tensão de $10 \mathrm{kV}$ para comparar o tamanho médio dos poros obtidos pelos dois métodos.

\section{Resultados e Discussão}

A Figura 2 mostra os valores da altura da ascensão capilar do solvente no substrato ao quadrado $\left(\mathrm{h}^{2}\right)$ em função do tempo para o n-hexano para os dois tipos de filtros e a membrana interna da máscara. Em $23 \pm 1{ }^{\circ} \mathrm{C}$ n-hexano ${ }^{3}$ apresenta $\gamma_{\mathrm{L} / \mathrm{v}}$ e $\eta$ de $17,89 \times 10^{-3} \mathrm{~N} / \mathrm{m}$ e $0,300 \times 10^{-3} \mathrm{~Pa}$.s, respectivamente. Como o sistema é aberto e o n-hexano é volátil ${ }^{3}$ ( $\mathrm{T}_{\text {ebulição }} 69^{\circ} \mathrm{C}$ ), a evaporação do $\mathrm{n}$-hexano ao longo dos 90 s poderia abaixar a temperatura local. Por isso, algumas medidas foram feitas com n-dodecano ${ }^{3}$ que também possui baixa $\gamma_{\mathrm{L} / \mathrm{V}}\left(17,89 \times 10^{-3} \mathrm{~N} / \mathrm{m}\right)$ e $\eta\left(1,383 \times 10^{-3} \mathrm{~Pa} . \mathrm{s}\right)$, mas é menos volátil ${ }^{3}$ ( $\mathrm{T}_{\text {ebulição }}$ $216,2{ }^{\circ} \mathrm{C}$ ). Os resultados experimentais obtidos com n-hexano e n-dodecano apresentaram diferenças menores que $10 \%$. Desta forma, somente os resultados com n-hexano serão apresentados e discutidos. A variação de $\mathrm{h}^{2}$ com $\mathrm{t}$ foi linear para os dois tipos de papel de filtro. A partir dos valores de inclinação das retas foram estimados os valores de raio médio dos poros para os papéis de filtro de gramaturas $250 \mathrm{~g} / \mathrm{m}^{2}$ e $80 \mathrm{~g} / \mathrm{m}^{2}$ como sendo $0,195 \mu \mathrm{m} \pm 0,002 \mu \mathrm{m}$ e $0,084 \mu \mathrm{m} \pm 0,005 \mu \mathrm{m}$, respectivamente. A ascensão capilar na máscara apresentou um processo mais rápido até $30 \mathrm{~s}$ e um segundo estágio mais lento de $40 \mathrm{~s} \mathrm{a} 90 \mathrm{~s}$, evidenciado pelas inclinações das retas. A partir do processo mais rápido, foram obtidos valores de raio médio de $0,92 \pm 0,08 \mu \mathrm{m}$ e do processo mais lento, poros de tamanho médio de $0,236 \pm 0,019 \mu \mathrm{m}$. 


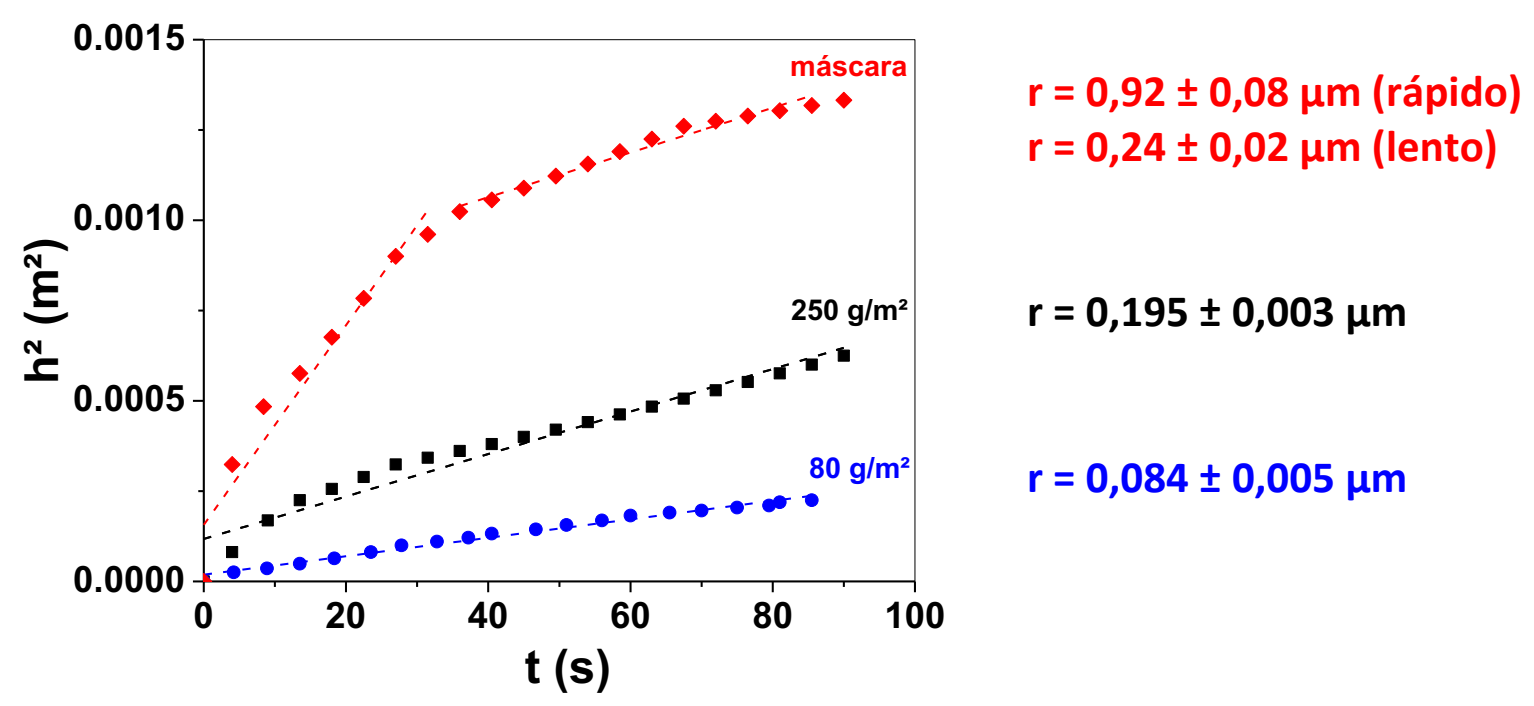

Figura 2. Valores de $\mathrm{h}^{2}\left(\mathrm{~m}^{2}\right)$ em função de $\mathrm{t}(\mathrm{s})$ para n-hexano no papel de filtro de $250 \mathrm{~g} / \mathrm{m}^{2}$ (símbolo preto), $80 \mathrm{~g} / \mathrm{m}^{2}$ (símbolo azul) e máscara (símbolo vermelho); valores de coeficientes angulares são $5.88 \times 10^{-6} \mathrm{~m}^{2} / \mathrm{s}$ $\left(250 \mathrm{~g} / \mathrm{m}^{2}\right), 2.56 \times 10^{-6} \mathrm{~m}^{2} / \mathrm{s}\left(80 \mathrm{~g} / \mathrm{m}^{2}\right)$ e para máscara, $2.76 \times 10^{-5} \mathrm{~m}^{2} / \mathrm{s}$ no estágio rápido e $6,18 \times 10^{-6} \mathrm{~m}^{2} / \mathrm{s}$ no estágio lento. Os valores de raio médio de poro estão indicados.

Os experimentos de ascensão capilar com água foram realizados para determinar o ângulo de contato $\theta$ entre água e o papel, para avaliar a molhabilidade dos materiais. A água não molhou a membrana interna da máscara porque é feita de polipropileno (PP), material muito hidrofóbico. Por outro lado, para os papéis de filtro de $250 \mathrm{~g} / \mathrm{m}^{2}$ e $80 \mathrm{~g} / \mathrm{m}^{2}$ foram determinados valores de $\cos \theta=0,987\left(\theta=9^{\circ}\right)$ e $\cos \theta=1,007\left(\theta \sim 0^{\circ}\right)$, respectivamente. Indicando que os papéis de filtro são altamente hidrofílicos, como esperado.

A Figura 3 mostra as imagens de MEV obtidas para os três materiais. As fibras celulósicas nas Figuras 3 (a) e (b) apresentam maior rugosidade, do que as fibras de PP (Figura 3c). As fibras de PP de espessura $\sim 10 \mu \mathrm{m}$ se sobrepõem formando uma malha com uma larga distribuição de tamanho de poros, muitos sendo micrométricos. Sabendo que o corona vírus ${ }^{4}$ tem dimensões de 50 a $200 \mathrm{~nm}$, é mais adequado adicionar mais uma camada de filtro (de papel, por exemplo) para que as fibras cruzadas criem poros menores e dificultem a passagem do vírus. Os valores de raio médio de poro determinados por ascensão capilar para a máscara com fibras de PP foram de 0,92 $\pm 0,08 \mu \mathrm{m}$ e 0,236 $\pm 0,019 \mu \mathrm{m}$, indicando que o método é sensível para uma faixa submicrométrica de poros. O papeis de filtro de $250 \mathrm{~g} / \mathrm{m}^{2}$ e $80 \mathrm{~g} / \mathrm{m}^{2}$ apresentam dimensões dos espaços entre as fibras bem variadas. $\mathrm{O}$ tamanho médio dos poros informado pelo fabricante é de $18 \mu \mathrm{m}$ e $26 \mu \mathrm{m}$, respectivamente. Esse tamanho médio indicado pelo fabricante é determinado por porômetro, aparelho que mede a pressão necessária para a primeira bolha de ar atravessar os poros ${ }^{5}$. A pressão é inversamente proporcional ao raio do poro (eq. 2). Os raios médios de poro determinados pelo método de Washburn com nhexano para os filtros $250 \mathrm{~g} / \mathrm{m}^{2}$ e $80 \mathrm{~g} / \mathrm{m}^{2}$ foram $0,195 \mu \mathrm{m} \pm 0,003 \mu \mathrm{m}$ e $0,084 \mu \mathrm{m} \pm 0,005 \mu \mathrm{m}$, respectivamente, e correspondem provavelmente ao tamanho de poros na estrutura das fibras celulósicas e não ao espaço vazio entre as fibras. 


\section{(a)}

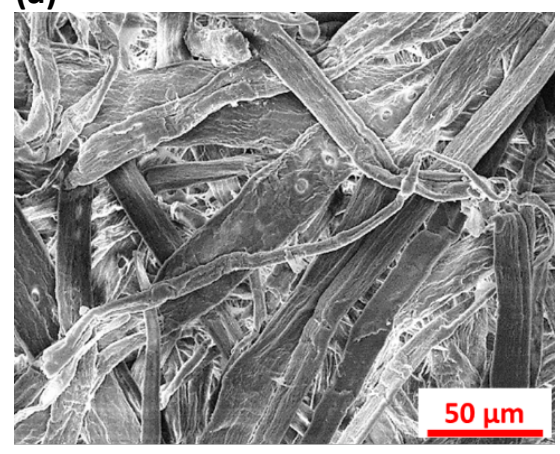

(b)

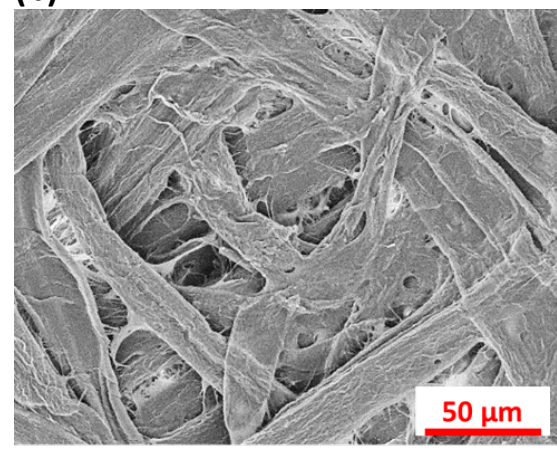

(c)

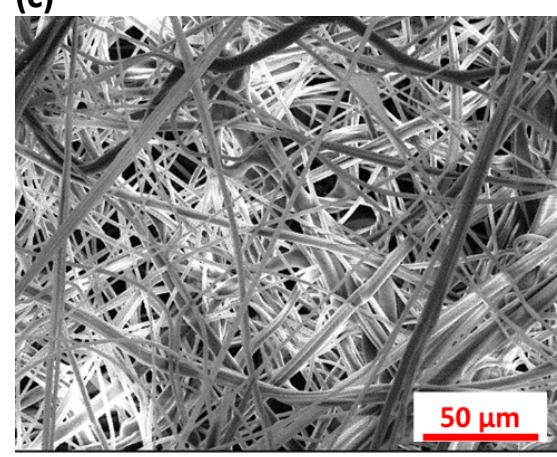

Figura 3. Imagens de MEV para filtro de papel com gramatura (a) $250 \mathrm{~g} / \mathrm{m}^{2}$ e (b) $80 \mathrm{~g} / \mathrm{m}^{2}$ e (c) membrana interna de polipropileno (PP) da máscara hospitalar.

\section{Conclusões}

A instrumentação de baixo custo ( US\$ 100) aliada à câmera do celular, se mostrou eficiente para medidas reprodutíveis de ângulo de contato de materiais celulósicos porosos. Os baixos valores de ângulo de contato encontrados para água em papéis de filtro são condizentes com a alta molhabilidade dos materiais pela água, mostrando que a instrumentação é confiável para medidas de molhabilidade de materiais porosos. Os tamanhos médios de poro determinados por ascensão capilar ficaram aproximadamente duas ordens de grandeza abaixo dos valores nominais determinados por porômetro. A principal diferença é que na ascensão capilar o n-hexano (ou n-dodecano) permeia livremente por todos os poros micrométricos e sub-micrométricos, enquanto no porômetro, ar comprimido é pressionado para atravessar o papel de filtro, o que faz com o que o ar procure os caminhos mais fáceis, ou seja, os maiores poros. As análises de MEV revelaram que os poros nos papéis de filtro e membrana interna da máscara são vazios formados pela sobreposição de fibras naturais ou sintéticas.

\section{Agradecimentos}

Agradecimento ao Programa Unificado de Bolsas da USP, Projeto 141-Desenvolvimento de novos experimentos para compreensão de equilíbrio químico e capilaridade. LMF e DFSP agradecem ao CNPq (171250/2017, 306848/2017 e 421014/2018-0) pelo suporte financeiro.

\section{Referências}

1. Kontogeorgis, G.M.; Kill, S. Introduction to Applied Colloid and Surface Chemistry, John Wiley \& Sons, Ltd., West Sussex, 2016.

2. Berg, J. C. An Introduction to Interfaces and Colloids: The Bridge to Nanoscience, 2010, World Scientific Publishing, Singapore, 2010.

3. West, R. C.; Astle, M.J.; Beyer, W.H. CRC Handbook of chemistry and physics. CRC Press, Inc. Boca Raton, Florida, 1985.

4. Chen, N. et al. Lancet 2020, 395, 507-513.

5. Norma técnica ASTM F316-03 1762019. 